\title{
Endocarditis derecha aislada en pacientes no adictos a drogas por vía parenteral
}

\author{
Ana Revillaa , Javier López ${ }^{a}$, Eduardo Villacortaa, Itziar Gómezaa, Teresa Sevillaa , Miguel Ángel del Pozob, \\ Luis de la Fuente ${ }^{a}$, María del Carmen Manzanoc, Pedro Mota ${ }^{a}$, Santiago Flórez ${ }^{a}$, Isidre Vilacostac, \\ Cristina Sarriád, Mariano Sánchez ${ }^{\mathrm{e}}$ y José Alberto San Román ${ }^{\mathrm{a}}$
}

alnstituto de Ciencias del Corazón (ICICOR). Hospital Clínico. Valladolid. España.

${ }^{b}$ Departamento de Enfermedades Infecciosas. Hospital Clínico. Valladolid. España.

'Departamento de Cardiología. Hospital Clínico San Carlos. Madrid. España.

${ }^{d}$ Departamento de Medicina Interna. Hospital de La Princesa. Madrid. España.

eInstituto de Biología y Genética Molecular (IBGM). CSIC. Valladolid. España.

Introducción y objetivos. La endocarditis derecha aislada es una entidad poco conocida cuando afecta a pacientes no portadores de marcapasos y no adictos a drogas por vía parenteral (ADVP). Nuestro objetivo es estudiar la frecuencia actual de esta entidad y describir su perfil clínico, microbiológico, ecocardiográfico y pronóstico.

Métodos. Hemos analizado 17 casos de endocarditis derecha aislada en pacientes no ADVP y no portadores de marcapasos, de un total de 583 episodios consecutivamente diagnosticados de endocarditis infecciosa (3\%).

Resultados. La media de edad fue $38 \pm 15$ años y 11 pacientes eran varones. El $47 \%$ de los pacientes presentaban alguna enfermedad predisponente. El catéter intravascular fue la puerta de entrada más frecuente (35\%). Los síntomas y signos más comunes al ingreso fueron fiebre, disnea, embolia pulmonar séptica, derrame pleural e insuficiencia cardiaca derecha. El microorganismo más frecuente fue Staphylococcus aureus (41\%). La mayoría de los casos asentaron en la válvula tricúspide (82\%). La embolia pulmonar recurrente fue la complicación más frecuente y la principal causa de cirugía, que fue precisa en $5(29 \%)$ casos. Fallecieron $2(12 \%)$ pacientes, ambos por shock séptico. En el seguimiento, 1 paciente falleció al mes del alta por causa desconocida y 1 presentó una recaída al tercer mes.

Conclusiones. La endocarditis derecha aislada debe incluirse en el diagnóstico diferencial de pacientes con síndrome febril, síntomas respiratorios y alguna enfermedad predisponente, aunque no sean portadores de marcapasos o ADVP. La presencia de catéteres intravascula-

Este estudio fue financiado en parte por la Red Cooperativa de Investigación Cardiovascular (RECAVA) del Instituto de Salud Carlos III y por una beca de la Sociedad Castellano-Leonesa de Cardiología (SOCALEC).

Correspondencia: Dra. A. Revilla Orodea.

Departamento de Cardiología y Cirugía Cardíaca. Instituto de Ciencias del Corazón (ICICOR). Hospital Clínico Universitario.

Ramón y Cajal, 3-5. Valladolid. España.

Correo electrónico: arevillaorodea@gmail.com

Recibido el 15 de abril de 2008

Aceptado para su publicación el 16 de septiembre de 2008. res y la bacteriemia por estafilococos refuerzan la sospecha de endocarditis en estos pacientes.

Palabras clave: Endocarditis derecha. Embolia pulmonar. Staphylococcus aureus. Insuficiencia cardiaca derecha.

\section{Isolated Right-Sided Valvular Endocarditis in Non-Intravenous Drug Users}

Introduction and objectives. Characteristics of isolated right-sided endocarditis in patients without a pacemaker and who are not intravenous drug users (IVDU) are poorly understood. The aim of this study was to investigate the current frequency of this entity and describe its clinical, microbiological, echocardiographic and prognostic profile.

Methods. We have prospectively analyzed 17 consecutive cases of isolated right-sided endocarditis in non-IVDU who did not have a pacemaker, out of a total of 583 consecutive episodes of endocarditis (2.9\%).

Results. Mean age was $38 \pm 15$ years; 11 of the 17 patients were men. Almost half of the patients had at least one predisposing disease. An intravascular catheter was the most frequent port of entry (35\%). The most common signs and symptoms on admission were fever, dyspnea, septic pulmonary embolisms, pleural effusion and right-sided heart failure. The most frequent microorganism was Staphylococcus aureus (41\%). In most cases (82\%) the infection was located in the tricuspid valve. Recurrent pulmonary embolisms were the most frequent complication and the main cause for surgery, which was needed in 5 patients (29\%). Two patients died during hospitalization (12\%), both from septic shock. During follow-up one patient died of unknown causes 1 month after discharge, and other relapsed 3 months after discharge.

Conclusions. Isolated right-sided endocarditis should be included in the differential diagnosis of patients with febrile syndrome, respiratory symptoms and predisposing disease, even when they do not have a pacemaker and are not IVDU. The presence of intravascular catheters and Staphylococcus bacteriemia should heighten suspicion of endocarditis. 
Key words: Right-sided endocarditis. Pulmonary embolisms. Staphylococcus aureus. Right-sided heart failure.

Full English text available from: www.revespcardiol.org

\section{ABREVIATURAS}

ADVP: adicto a drogas por vía parenteral.

\section{INTRODUCCIÓN}

La endocarditis derecha es una entidad clínica bien definida en pacientes con antecedentes de adicción a drogas por vía parenteral (ADVP) o portadores de marcapasos u otros dispositivos intracardiacos ${ }^{1-4}$. Su forma de presentación clínica y su tratamiento difieren de los de la endocarditis izquierda ${ }^{1-4}$, y su pronóstico es más favorable, puesto que muchos pacientes pueden alcanzar una curación con tan sólo tratamiento médico ${ }^{1,4} \mathrm{o}$ con la extracción quirúrgica del dispositivo en la endocarditis por marcapasos ${ }^{2-3}$. La fiebre asociada a antecedentes de ADVP o de uso de marcapasos debe hacer sospechar una endocarditis, que puede confirmarse mediante hemocultivos y ecocardiografía.

La endocarditis derecha aislada, en ausencia de antecedentes de ADVP o en pacientes no portadores de marcapasos, es una entidad poco conocida. La mayor parte de los estudios publicados sobre esta enfermedad corresponden a descripciones antiguas de casos aisla$\operatorname{dos}^{5-7}$. La serie más amplia, descrita por Nandakumar et $\mathrm{al}^{8}$, resumió las características de 29 casos de endocarditis tricuspídea en pacientes sin ADVP publicados por diversos autores las últimas décadas del siglo pasado. El estudio de Naidoo ${ }^{9}$ analizó 15 casos de endocarditis derecha en pacientes sin ADVP; sin embargo, se trata de un análisis retrospectivo en el que se incluyeron también casos de endocarditis izquierda.

Así pues, carecemos de información sobre el perfil clínico y el pronóstico de la endocarditis derecha en pacientes no portadores de marcapasos y sin antecedentes de ADVP. El objetivo de este estudio fue investigar la frecuencia actual de esta entidad y describir su perfil clínico, microbiológico, ecocardiográfico y pronóstico.

\section{MÉTODOS}

Analizamos 583 episodios de endocarditis diagnosticados de manera consecutiva entre 1996 y 2006 en las unidades de cardiología y cirugía cardiaca de cuatro centros terciarios, siguiendo los criterios de Duke (hasta 2002) o los criterios de Duke modificados ${ }^{10}$ (de 2003 a 2006). De los 93 (16\%) episodios localizados en el lado derecho del corazón, 44 (47\%) afectaron a un dispositivo intracardiaco, $32(35 \%)$ a pacientes con ADVP y $17(18 \%)$ a pacientes sin antecedentes de ADVP que no eran portadores de marcapasos. Estos 17 pacientes (el 2,9\% de la cohorte total de nuestra serie) constituyen el grupo de estudio para este trabajo. En la figura 1 se muestra la distribución de nuestra población de pacientes.

Para cada paciente utilizamos el mismo protocolo para registrar la información de manera prospectiva, empezando el día del ingreso en el hospital. El protocolo incluía 90 variables ${ }^{11}$ : 19 epidemiológicas, 8 clínicas, 10 analíticas, 4 radiográficas, 6 electrocardiográficas, 13 microbiológicas, 16 ecocardiográficas y 14 características de seguimiento. De todos los pacientes, registramos los resultados de al menos una exploración física, un electrocardiograma, una radiografía de tórax, un análisis de orina, tres hemocultivos al ingreso, tres hemocultivos $48 \mathrm{~h}$ después de iniciado el tratamiento antibiótico y una ecocardiografía transtorácica y transesofágica. Se inició tratamiento antibiótico empírico tras la obtención de los hemocultivos si el

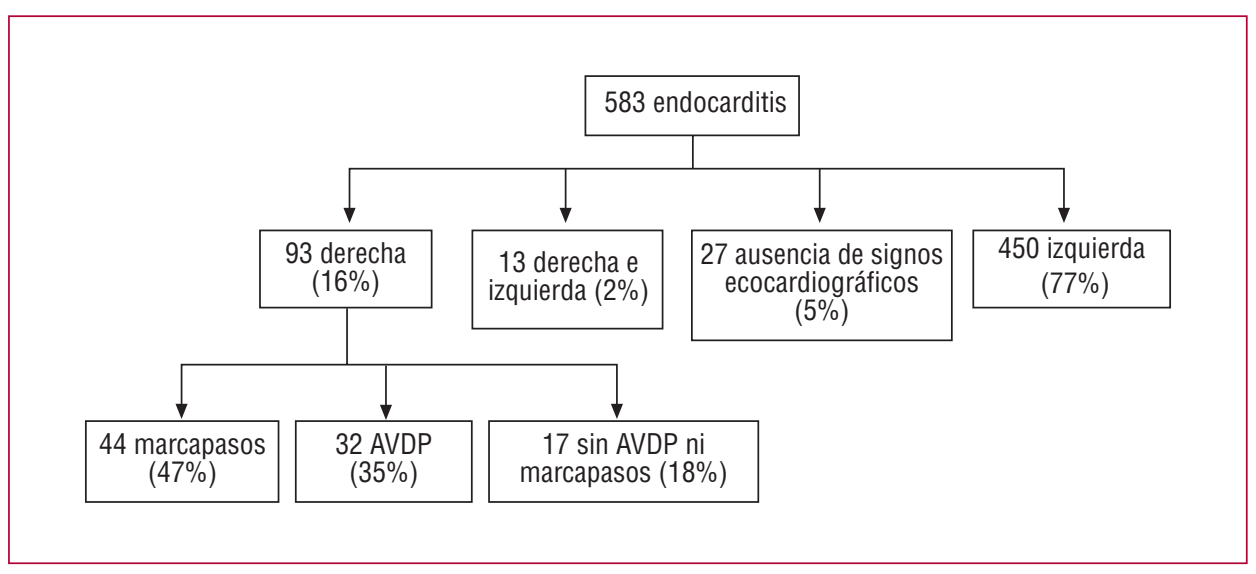

Fig. 1. Distribución de nuestra población. ADVP: adictos a drogas por vía parenteral. 
estado clínico del paciente así lo requería, y se modificó el tratamiento en caso necesario en función de los resultados de los hemocultivos y el antibiograma. Si los hemocultivos obtenidos a las $72 \mathrm{~h}$ eran negativos, se realizaban pruebas serológicas para Chlamydia, Brucella, fiebre Q, Legionella y Mycoplasma.

\section{Definición de los términos}

La endocarditis infecciosa asociada a la asistencia sanitaria incluye la infección nosocomial y la infección asociada a la asistencia sanitaria no nosocomial. La endocarditis se consideró nocosomial cuando se producía en un paciente hospitalizado durante más de $72 \mathrm{~h}$ antes de la aparición de signos o síntomas indicativos de endocarditis. La endocarditis infecciosa asociada a la asistencia sanitaria no nosocomial se definió como la aparecida en pacientes ambulatorios con un contacto amplio con la asistencia sanitaria (dispositivos permanentes para tratamiento intravenoso, catéteres de hemodiálisis, etc.). Los episodios diagnosticados en el momento del ingreso (o en las primeras $72 \mathrm{~h}$ siguientes al ingreso) en pacientes que no cumplían los criterios de infección asociada a la asistencia sanitaria se consideraron contraídos en la comunidad.

Consideramos endocarditis protésica precoz como la producida en el plazo de 1 año tras la intervención quirúrgica $^{12}$. La enfermedad se consideró de inicio agudo cuando el tiempo transcurrido entre la aparición de los síntomas y el ingreso no era superior a 15 días. La insuficiencia renal se definió como una concentración sérica de creatinina $>2 \mathrm{mg} / \mathrm{dl}$. La anemia crónica se definió como una concentración de hemoglobina < $9 \mathrm{~g} / \mathrm{dl}$ documentada durante al menos 1 año antes de la hospitalización. Consideramos que cualquier tratamiento que pudiera reducir la inmunidad celular o humoral constituía un tratamiento inmunosupresor. Los criterios ecocardiográficos para vegetaciones, abscesos, seudoaneurismas y fístulas han sido descritos previamente ${ }^{13}$.

Las indicaciones de cirugía fueron la insuficiencia cardiaca resistente al tratamiento médico, la endocarditis fúngica, la embolia recurrente con vegetaciones persistentes en la ecocardiografía y la infección no controlada, definida como bacteriemia persistente o fiebre de más de 7 días de duración a pesar del tratamiento antibiótico apropiado, una vez descartados otros focos de infección.

\section{Análisis estadístico}

Presentamos un análisis descriptivo de las variables epidemiológicas, analíticas, radiográficas, electrocardiográficas, microbiológicas, ecocardiográficas y de seguimiento en 17 pacientes con ADVP y no portadores de marcapasos que presentaban una endocarditis derecha aislada. Las variables continuas se expresan en forma de media \pm desviación estándar o mediana [rango intercuartílico], y las variables discretas se presentan con el valor absoluto y el porcentaje. Todos los datos se analizaron con el programa SPSS, v. 12.0 (SPSS Inc., Chicago, Illinois, Estados Unidos).

\section{RESULTADOS}

\section{Hallazgos epidemiológicos y clínicos}

La media de edad fue $38 \pm 15$ (20-61) años; 11 pacientes eran varones. La infección fue nosocomial en 7 pacientes, contraída en la comunidad en 7 y asociada a la asistencia sanitaria no nosocomial en 3. La puerta de entrada pudo determinarse en 11 pacientes y fue la siguiente: catéter intravascular en 6 casos, intervenciones genitourinarias (legrado por aborto séptico) en 3, cirugía cardiaca previa (sustitución de válvula aórtica) en 1 y derrame articular séptico en el hombro en 1 . Se documentaron antecedentes de enfermedades cardiacas en 4 pacientes: 2 de ellos eran portadores de prótesis valvulares (1 prótesis mecánica aórtica y 1 prótesis biológica de válvula tricúspide) y 2 presentaban cardiopatías congénitas (comunicación interventricular en 1 caso y tétrada de Fallot en el otro); 3 pacientes tenían antecedentes de un episodio previo de endocarditis ( 1 endocarditis aórtica y 2 endocarditis tricuspídeas). Se identificaron enfermedades o trastornos predisponentes en 8 (47\%) pacientes: inmunodepresión en 4, anemia crónica en 3 , diabetes mellitus en 3 , tratamiento inmunosupresor en 3 , insuficiencia renal crónica en 3 y cáncer en 3 . En 5 pacientes había más de una enfermedad predisponente. En la mayor parte de los pacientes, el inicio de la enfermedad fue agudo, y tan sólo 2 pacientes presentaban síntomas de más de 2 meses de evolución. En 13 (76\%) episodios, el paciente había recibido tratamiento antibiótico antes del diagnóstico de endocarditis para tratar un síndrome febril que generalmente se atribuyó a una infección respiratoria.

Los síntomas y signos más comunes al ingreso fueron fiebre $(n=15)$ y disnea $(n=5)$. Tres pacientes fueron ingresados en shock séptico, y otros 3 presentaban signos clínicos de insuficiencia cardiaca derecha. Las radiografías de tórax mostraron cardiomegalia en 9 pacientes, embolia pulmonar séptica en 7 , derrame pleural en 6 e insuficiencia cardiaca izquierda en 2.

\section{Hallazgos microbiológicos}

Los hemocultivos fueron positivos al ingreso en 14 pacientes, y se positivizaron en las primeras $48 \mathrm{~h}$ en 1 paciente. En 2 pacientes no pudo identificarse el microorganismo causal; ambos estaban recibiendo tratamiento antibiótico cuando se obtuvieron los hemocultivos. Tras $48 \mathrm{~h}$ de tratamiento antibiótico, los hemocultivos se negativizaron en 13 pacientes. El 
TABLA 1. Resumen de características clínicas y evolución de nuestros pacientes

\begin{tabular}{|c|c|c|c|c|c|c|c|c|}
\hline Paciente & Edad & Sexo & Enfermedad predisponente & Puerta de entrada & Microorganismo causal & Complicaciones & Cirugía & Muerte hospitalaria \\
\hline 1 & 38 & Mujer & 0 & Aborto séptico & MSSA & EP recurrente & + & 0 \\
\hline 2 & 54 & Mujer & $\begin{array}{l}\text { Anemia crónica, } \\
\text { IR crónica }\end{array}$ & Catéter intravascular & MSSA & EP, BAV completo & 0 & 0 \\
\hline 3 & 54 & Varón & 0 & $\begin{array}{c}\text { Sustitución valvular } \\
\text { aórtica }\end{array}$ & MRSA & 0 & 0 & 0 \\
\hline 4 & 22 & Varón & 0 & Catéter intravascular & MRSA & IR de novo, EP & 0 & 0 \\
\hline 5 & 33 & Varón & IS & - & MSSA & IR de novo & 0 & 0 \\
\hline 6 & 20 & Mujer & 0 & Aborto séptico & Negativo & $\begin{array}{l}\text { ICD al ingreso, } \\
\text { EP recurrente }\end{array}$ & + & 0 \\
\hline 7 & 30 & Varón & 0 & - & Streptococcus bovis & IP & + & 0 \\
\hline 8 & 26 & Mujer & 0 & Aborto séptico & Negativo & EP recurrente & + & 0 \\
\hline 9 & 26 & Varón & IS, tIS, cáncer & Catéter intravascular & Candida albicans & 0 & 0 & 0 \\
\hline 10 & 58 & Varón & 0 & Infección local & MRSA & 0 & 0 & 0 \\
\hline 11 & 53 & Varón & DM & - & Klebsiella pneumoniae & 0 & 0 & 0 \\
\hline 12 & 53 & Mujer & 0 & - & $\begin{array}{c}\text { Streptococcus } \\
\text { agalactiae }\end{array}$ & SS al ingreso & 0 & + \\
\hline 13 & 22 & Varón & 0 & - & $\begin{array}{c}\text { Streptococcus } \\
\text { oralis }\end{array}$ & $\begin{array}{l}\text { ICD al ingreso, } \\
\text { IP, ICD resistente }\end{array}$ & + & 0 \\
\hline 14 & 61 & Varón & $\begin{array}{l}\text { Anemia crónica, } \\
\text { DM, cáncer }\end{array}$ & Catéter intravascular & CNS & $\begin{array}{l}\text { ICD al ingreso, } \\
\text { SS al ingreso }\end{array}$ & 0 & 0 \\
\hline 15 & 34 & Varón & IS, tIS, IR crónica & - & MSSA & 0 & 0 & 0 \\
\hline 16 & 39 & Varón & $\begin{array}{l}\text { IR crónica, IS, tIS, } \\
\text { DM, cáncer }\end{array}$ & Catéter intravascular & $\begin{array}{c}\text { Polimicrobiana } \\
\text { (CNS, Pseudomonas sp., } \\
\text { Candida albicans) }\end{array}$ & $\begin{array}{l}\text { ICI en evolución, } \\
\text { SS al ingreso }\end{array}$ & 0 & + \\
\hline 17 & 22 & Varón & Anemia crónica & Catéter intravascular & $\begin{array}{c}\text { Propionibacterium } \\
\text { acnes }\end{array}$ & EP & 0 & 0 \\
\hline
\end{tabular}

BAV: bloqueo auriculoventricular; CNS: Staphylococcus sp. coagulasa-negativo; DM: diabetes mellitus; EP: embolia pulmonar; ICD: insuficiencia cardiaca derecha; ICI: insuficiencia cardiaca izquierda; IP: infección persistente; IR: insuficiencia renal; IS: inmunodeficiencia; MRSA: Staphylococcus aureus resistente a meticilina; MSSA: Staphylococcus aureus sensible a meticilina; SS: shock séptico; tIS: tratamiento inmunosupresor.

microorganismo aislado con más frecuencia fue Staphylococcus aureus $(\mathrm{n}=7 ; 41 \%)$, sensible a meticilina en 4 pacientes y resistente a meticilina en 3 . En la tabla 1 se muestra el perfil microbiológico de nuestros 17 pacientes.

\section{Hallazgos ecocardiográficos}

La endocarditis asentó en la válvula tricúspide en 14 (82\%) pacientes. En los demás casos, afectó a la vena cava superior, a un defecto del tabique ventricular y una endocarditis precoz de una prótesis valvular biológica tricuspídea. Ninguno de los episodios afectó a más de una válvula. Todos los pacientes presentaban vegetaciones en la ecocardiografía transesofágica, y la mayoría de ellas eran grandes $-(13 \pm 7) \times(8 \pm 6)$ $\mathrm{mm}$; área, $87 \pm 65 \mathrm{~mm}^{2}$ - y muy móviles (fig. 2). Sin embargo, la aparición de complicaciones perivalvulares fue muy poco frecuente (3 fístulas). Se produjo una rotura valvular en 1 caso y una insuficiencia tricuspídea grave en 12 pacientes.

\section{Curso clínico}

Durante la hospitalización, en 1 paciente se desarrolló insuficiencia cardiaca izquierda (por sobrecarga hí- drica en un paciente en hemodiálisis y con una función ventricular izquierda reducida), 2 pacientes presentaron insuficiencia renal de nueva aparición y 1 paciente presentó bloqueo auriculoventricular completo.

La anemia fue frecuente en nuestros pacientes, y solamente 3 de ellos presentaban al ingreso una concentración de hemoglobina $>12 \mathrm{~g} / \mathrm{dl}$. En 10 pacientes, la concentración era $<10 \mathrm{~g} / \mathrm{dl}$, y en 7 el valor era $<9$ $\mathrm{g} / \mathrm{dl}$. La hematuria microscópica fue también frecuente y se objetivó en $8(44 \%)$ pacientes.

La cirugía cardiaca fue necesaria en 5 (29\%) pacientes: 3 a causa de embolia pulmonar recurrente a pesar del tratamiento apropiado, con persistencia de vegetaciones móviles grandes en la ecocardiografía, y 2 por una infección persistente (1 de ellos presentaba también insuficiencia cardiaca derecha resistente al tratamiento). La cirugía consistió en desbridamiento y reparación de la válvula tricúspide en 3 casos y en sustitución valvular en 2 (1 prótesis valvular mecánica y 1 biológica). En un paciente fue necesaria la implantación de un marcapasos epicárdico permanente a causa de un bloqueo auriculoventricular completo.

La mediana de duración de la hospitalización fue de 37 [30-63] días. Durante la hospitalización, fallecieron 2 pacientes, ambos por shock séptico. Un paciente falleció 24 h después del diagnóstico, tras una clínica de 
Fig. 2. Ecocardiografía transtorácica en un paciente con endocarditis tricuspídea. Vegetación de gran tamaño (flecha) que causa una severa insuficiencia valvular.

AD: aurícula derecha; Al: aurícula izquierda; VD: ventrículo derecho.

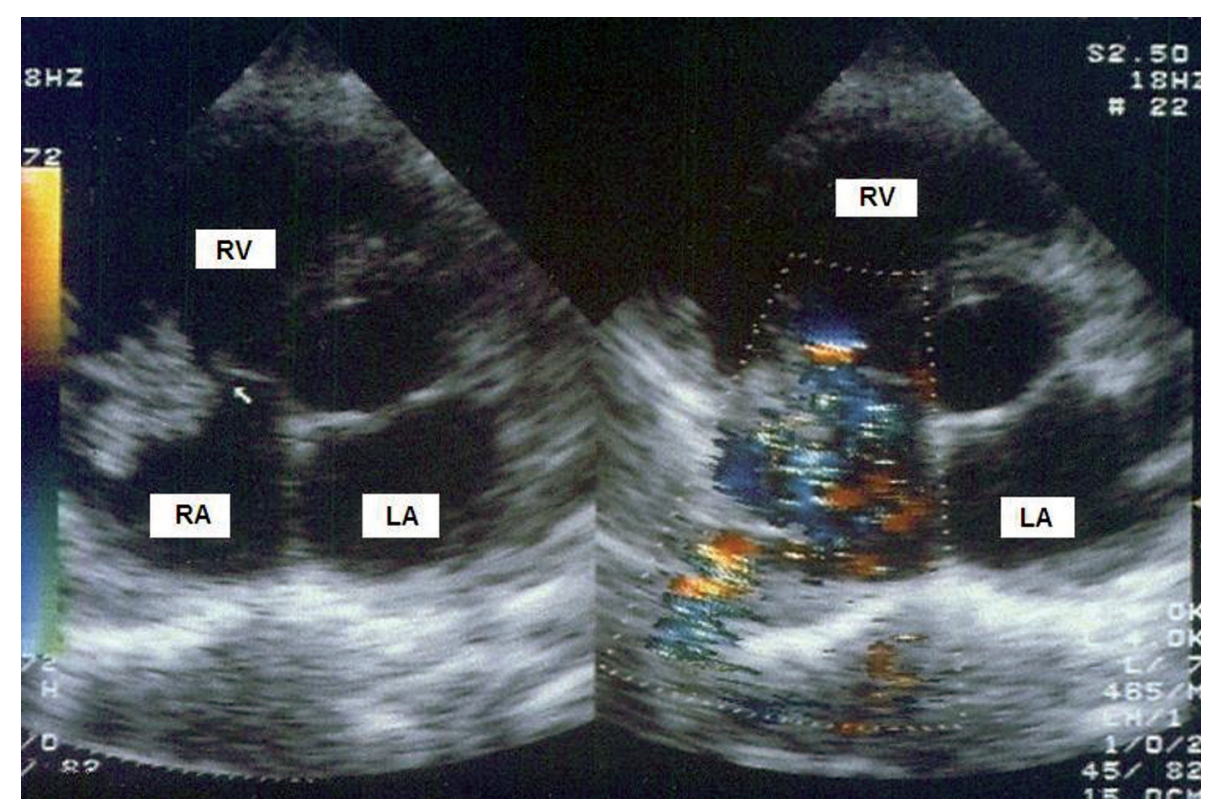

1 mes de síndrome febril que se trató sin éxito extrahospitalariamente. El otro paciente tenía un cáncer metastásico y una insuficiencia renal terminal, y refería un síndrome febril de 2 meses de evolución tratado sin éxito con antibióticos antes del diagnóstico. Así pues, la mortalidad intrahospitalaria fue del $12 \%$. Por lo que respecta al pronóstico, la mortalidad en pacientes sin ADVP y no portadores de marcapasos que presentaron una endocarditis derecha aislada fue inferior a la del grupo de endocarditis izquierda (el 11 frente al $33 \%$; $\mathrm{p}=0,06$ ).

El seguimiento posterior al alta en 11 pacientes tuvo una mediana de duración de 344 [180-1.227] días. Un paciente falleció 1 mes después del alta por causas desconocidas, y otro paciente con endocarditis tricuspídea por Propionibacterium acnes presentó una recaída al cabo de 3 meses; todos los demás pacientes se mantuvieron asintomáticos. En la tabla 1 se resumen las características clínicas de nuestros 17 pacientes.

\section{DISCUSIÓN}

La endocarditis derecha se asocia a menudo a los dispositivos intracardiacos o a antecedentes de ADVP, $\mathrm{y}$ en muchos estudios publicados se han descrito estas dos formas de presentación de la enfermedad ${ }^{1-4}$. Sin embargo, la aparición de una endocarditis derecha en un paciente que no presenta ninguno de estos dos antecedentes se ha descrito con muy poca frecuencia, probablemente por la baja incidencia de la enfermedad. De hecho, nuestra búsqueda bibliográfica identificó solamente descripciones antiguas de casos aislados ${ }^{5-9}$. En este estudio se presentan los resultados de la pri- mera serie prospectiva publicada hasta la fecha de pacientes con endocarditis derecha sin ADVP y no portadores de marcapasos.

Aunque la frecuencia de la endocarditis derecha en el tipo de pacientes que se describe aquí fue baja en relación con el conjunto del grupo de pacientes con endocarditis (el 18\% del total de pacientes con endocarditis derecha, el 3\% del total de pacientes con endocarditis de nuestra serie), la frecuencia fue superior a la inicialmente esperada, sobre todo teniendo en cuenta que más de la mitad de los casos se dieron en pacientes aparentemente sanos, sin enfermedades predisponentes. Es probable que la frecuencia real sea superior a la que nosotros observamos, por dos razones. Los síntomas iniciales de una endocarditis derecha son similares a los de una infección respiratoria (fiebre, disnea e infiltrado pulmonar), y ello hace probable la confusión diagnóstica. Además, dado que el estado clínico del paciente mejora con el tratamiento antibiótico, muchos casos de endocarditis derecha quedan sin diagnosticar. Esto explica que la mayor parte de los pacientes de nuestra serie recibieran tratamiento antibiótico antes de que se estableciera el diagnóstico correcto. Por otra parte, la incidencia real de la enfermedad puede haberse infravalorado, ya que la mayor parte de los estudios han incluido a pacientes con endocarditis complejas o pacientes en los que la enfermedad tiene una mala evolución clínica y por ello son remitidos a centros de referencia ${ }^{1,8}$. Esto puede comportar un sesgo de selección, de manera que nuestras conclusiones sólo serían aplicables a poblaciones similares a la nuestra.

En general, la endocarditis derecha aislada en pacientes sin ADVP y no portadores de marcapasos afec- 
ta a individuos jóvenes ${ }^{9,14}$. La presencia de uno o varios factores predisponentes (inmunodeficiencia, insuficiencia renal o cáncer) no es infrecuente. En estos pacientes, la fiebre no suele indicar un diagnóstico de endocarditis, sino que apunta más bien hacia otros tipos de infección (p. ej., respiratoria o abdominal) y ello puede retrasar aún más el diagnóstico. Así pues, la endocarditis derecha debe incluirse en el diagnóstico diferencial de los pacientes jóvenes con fiebre y una enfermedad predisponente.

En la mayor parte de los casos pudo identificarse la puerta de entrada de la infección. La más frecuente fue un catéter intravascular, y de hecho la posibilidad de aparición de una endocarditis a causa de un catéter venoso central se había señalado ya con anterioridad ${ }^{15}$. Los catéteres vasculares son el principal origen de bacteriemias, en especial de las infecciones causadas por estafilococos ${ }^{16}$, que fueron los microorganismos hallados con mayor frecuencia en nuestra serie.

El retraso en el diagnóstico se debe, en parte, al hecho de que los criterios de Duke no son apropiados para los pacientes con endocarditis derecha (es decir, la sensibilidad de estos criterios para detectar la enfermedad es baja), y según muchos autores es preciso modificarlos ${ }^{17,18}$. Se ha propuesto una serie de criterios mayores y menores para el diagnóstico de la endocarditis derecha ${ }^{17}$. Ésta no se manifiesta clínicamente con los signos clásicos de la endocarditis izquierda ${ }^{5}$, sino con síntomas respiratorios. Es frecuente que la radiografía de tórax muestre embolia séptica y derrame pleural. Dado que predominan los signos y síntomas respiratorios, es frecuente que se diagnostique a los pacientes una neumonía ${ }^{5,8}$. La combinación de infiltrados pulmonares y enfermedad renal puede motivar una sospecha de vasculitis ${ }^{8}$. La embolia pulmonar es frecuente en los pacientes con endocarditis derecha y podría retrasar el diagnóstico de la enfermedad si se interpreta como una causa de la endocarditis en vez de una manifestación de ella. La fiebre también es un signo frecuente en los primeros días siguientes a una embolia pulmonar, pero rara vez es muy alta o prolongada. Así pues, en los pacientes con embolia pulmonar y fiebre alta, mantenida o recurrente o con elevados valores de marcadores inflamatorios, deben descartarse otras etiologías del síndrome febril, como la endocarditis derecha, sobre todo si tienen factores de riesgo (catéteres venosos centrales, alcoholismo, cáncer, cardiopatía previa, etc. $)^{19}$.

Se ha señalado que debe sospecharse una endocarditis derecha en presencia del denominado «síndrome tricuspídeo»: episodios respiratorios recurrentes, anemia y hematuria microscópica ${ }^{8}$. El presente estudio corrobora esta asociación, puesto que el $24 \%$ de nuestros pacientes presentaba el síndrome tricuspídeo y el $65 \%$ tenía al menos dos de los tres componentes. La aparición de alteraciones renales en pacientes con endocarditis infecciosa es frecuente, y estas alteraciones pueden manifestarse en forma de diversas complicaciones con una distinta trascendencia clínica. La manifestación renal más frecuente es la hematuria o la proteinuria (o ambas), y la observación anatomopatológica más frecuente es la glomerulonefritis, que suele ser proliferativa y difusa o vasculítica ${ }^{20,21}$.

Los hemocultivos fueron positivos en la mayor parte de los pacientes. En nuestra serie, el microorganismo causal más frecuente fue $S$. aureus, igual que se había descrito en series anteriores ${ }^{8,9}$. La bacteriemia estafilocócica se asocia con mayor frecuencia a endocarditis que a neumonía ${ }^{5}$, por lo que la identificación de este germen aumenta la necesidad de descartar la endocarditis, en especial en pacientes con enfermedades predisponentes o portadores de un catéter intravascular. Es interesante señalar que rara vez se identificaron hongos como agentes causales, a pesar de que la mayor parte de nuestros pacientes habían recibido tratamiento antibiótico previo, estaban inmunodeficientes o eran portadores de catéteres intravasculares. Es de destacar la ausencia de Streptococcus viridans, al igual que en un estudio anterior ${ }^{8}$. Es probable que los casos de endocarditis derecha que no habían sido diagnosticados por haberlos confundido con infecciones respiratorias (y haber mejorado con el tratamiento antibiótico) fueran causados por gérmenes poco agresivos, como los estreptococos. Esto explicaría que Staphylococcus aureus sea el microorganismo más común en nuestra serie, puesto que las infecciones causadas por los gérmenes más agresivos sólo se diagnostican cuando el paciente no mejora al recibir tratamiento para una neumonía.

Las vegetaciones fueron una observación constante en nuestros pacientes. Además, éstas eran grandes como consecuencia de la menor presión ejercida por las cámaras cardiacas derechas, que permite que las vegetaciones crezcan más que en el lado izquierdo ${ }^{22}$. Muchos estudios han relacionado las vegetaciones grandes y móviles con una incidencia elevada de embolias $^{23,24}$, y ello puede explicar la presencia frecuente de embolias pulmonares en nuestra serie.

En la mayor parte de nuestros pacientes, el curso clínico fue satisfactorio con el tratamiento médico solo. La cirugía cardiaca fue necesaria en el $29 \%$ de los pacientes, porcentaje inferior al descrito en series anteriores ${ }^{8}$, probablemente a causa de las mejoras en el tratamiento antibiótico en las últimas décadas. Las causas de la cirugía fueron similares a las de la endocarditis derecha en general ${ }^{1,5,8,9}$. Todos los pacientes quirúrgicos tuvieron un curso clínico satisfactorio. A diferencia de lo que ocurre en los pacientes con ADVP, la reinfección de la prótesis valvular no es un problema frecuente en los pacientes sin $\mathrm{ADVP}^{2}$. Solamente 1 de nuestros pacientes presentó una recaída al cabo de 3 meses. La mortalidad causada por la enfermedad fue baja e inferior a la asociada a la endocarditis izquierda, si bien la comparación no alcanzó signi- 
ficación estadística, probablemente por la diferencia de tamaño muestral entre los dos grupos.

\section{CONCLUSIONES}

En resumen, nuestros resultados indican que la endocarditis derecha aislada debe incluirse en el diagnóstico diferencial de los pacientes con síndrome febril, síntomas respiratorios y una enfermedad predisponente (cáncer, inmunodeficiencia o insuficiencia renal crónica), incluso cuando no sean portadores de marcapasos y no tengan antecedentes de ADVP. La presencia de un catéter intravascular, los antecedentes recientes de una intervención quirúrgica y la bacteriemia por estafilococos deben reforzar la sospecha de endocarditis derecha. El pronóstico parece ser más favorable que el de la endocarditis izquierda.

\section{BIBLIOGRAFÍA}

1. Moss R, Munt B. Injection drug use and right sided endocarditis. Heart. 2003;89:577-81.

2. López R, Rodríguez M, Hermida A, Lado FL. Endocarditis del marcapasos. An Med Interna. 2006;23:187-92.

3. Karchmer AW, Longworth DL. Infections of intracardiac devices. Cardiol Clin. 2003;21:253-71.

4. De Alarcón A, Villanueva JL. Endocarditis en adictos a drogas por vía parenteral. Endocarditis derecha. Influencia de la situación VIH. Rev Esp Cardiol. 1998;51 Supl 2:71-8.

5. Edmond JJ, Eykyn SJ, Smith LD. Community acquired staphylococcal pulmonary valve endocarditis in non-drug users: case report and review of the literature. Heart. 2001;86:E17.

6. Clifford CP, Eykyn SJ, Oakley CM. Staphylococcal tricuspid valve endocarditis in patients with structurally normal hearts and no evidence of narcotic abuse. QJM. 1994;87:755-7.

7. Roberts WC, Buchbinder NA. Right-sided valvular endocarditis: a clinicopathologic study of twelve necropsy patients. Am J Med. 1972;53:7-19.

8. Nandakumar R, Raju G. Isolated tricuspid valve endocarditis in nonaddicted patients: a diagnostic challenge. Am J Med Sci. 1997;314:207-12.

9. Naidoo DP. Right-sided endocarditis in the non-drug addict. Postgrad Med J. 1993;69:615-20.
10. Li JS, Sexton DJ, Mick N, Nettles R, Fowler WG Jr, Ryan T, et al. Proposed modifications to the Duke criteria for the diagnosis of infective endocarditis. Clin Infect Dis. 2000;30:633-8.

11. Revilla A, López J, Vilacosta I, Villacorta E, Rollán MJ, Echevarría JR, et al. Clinical and prognostic profile of patients with infective endocarditis who need urgent surgery. Eur Heart J. 2007;28:65-71.

12. López J, Revilla A, Vilacosta I, Villacorta E, González-Juanatey C, Gómez I, et al. Definition, clinical profile, microbiological spectrum, and prognostic factors of early-onset prosthetic valve endocarditis. Eur Heart J. 2007;28:760-5.

13. Graupner C, Vilacosta I, San Román J, Ronderos R, Sarriá C, Fernández $\mathrm{C}$, et al. Periannular extension of infective endocarditis. J Am Coll Cardiol. 2002;39:1204-11.

14. Ruiz M, Anguita M, Zayas R, Tejero I, Torres F, Giménez D, et al. Endocarditis infecciosa en pacientes no drogadictos sin cardiopatía predisponente. Características diferenciales. Rev Esp Cardiol. 1994;47:518-22.

15. Tsao MM, Katz D. Central venous catheter-induced endocarditis: human correlate of the animal experimental model of endocarditis. Rev Infect Dis. 1984;6:783-90.

16. Terpenning MS, Buggy BP, Kauffman CA. Hospital acquired infective endocarditis. Arch Intern Med. 1988;148:1601-3.

17. Robbins MJ, Frater RWM, Soeiro R, Frishman WH, Strom JA. Influence of vegetation size on clinical outcome of right-sided infective endocarditis. Am J Med. 1986;80:165-71.

18. Habib G, Derumeaux G, Avierinos JF, Casalta JP, Jamal F, Volot $\mathrm{F}$, et al. Value and limitations of the Duke criteria for the diagnosis of infective endocarditis. J Am Coll Cardiol. 1999;33:2023-9.

19. Nucifora G, Badano L, Hysko F, Allocca G, Gianfagna P, Fioretti P. Pulmonary embolism and fever. When should right-sided infective endocarditis be considered? Circulation. 2007;115:e1736.

20. Sadikoglu B, Bilge I, Kilicaslan I, Gokce MG, Emre S, Ertugrul T. Crescentic glomerulonephritis in a child with infective endocarditis. Pediatr Nephrol. 2006;21:867-9.

21. Majumdar A, Chowdhary S, Ferreira MA, Hammond LA, Howie $\mathrm{Aj}$, Lipkin GW, et al. Renal pathological findings in infective endocarditis. Nephrol Dial Transplant. 2000;15:1782-7.

22. San Román JA, Vilacosta I, Zamorano JL, Almería C, SánchezHarguindey L. Transesophageal echocardiography in right-sided endocarditis. J Am Coll Cardiol. 1993;21:1226-30.

23. Di Salvo G, Habib G, Pergola V, Avierinos JF, Philip E, Casalta JP, et al. Echocardiography predicts embolic events in infective endocarditis. J Am Coll Cardiol. 2001;37:1069-76.

24. Tischler MD, Vaitkus PT. The ability of vegetation size on echocardiography to predict clinical complications: A metaanalysis. J Am Soc Echocardiogr. 1997;10:562-8. 